\title{
Identification of NF-KB and PLCL2 as new susceptibility genes and highlights on a potential role of IRF8 through interferon signature modulation in systemic sclerosis
}

Maria Arismendi ${ }^{1,2}$, Matthieu Giraud ${ }^{1}$, Nadira Ruzehaji ${ }^{1}$, Philippe Dieudé3 ${ }^{3,4}$, Eugenie Koumakis ${ }^{1}$, Barbara Ruiz ${ }^{1}$, Paolo Airo ${ }^{5}$, Daniele Cusi ${ }^{6}$, Marco Matucci-Cerinic ${ }^{7}$, Erika Salvi ${ }^{6}$, Giovanna Cuomo ${ }^{8}$, Eric Hachulla ${ }^{9}$, Elisabeth Diot ${ }^{10}$, Paola Caramaschi ${ }^{11}$, Valeria Riccieri ${ }^{12}$, Jérôme Avouac ${ }^{1,13}$, Cristiane Kayser ${ }^{14}$ and Yannick Allanore ${ }^{1,13^{*}}$

\begin{abstract}
Introduction: Systemic sclerosis (SSC) and primary biliary cirrhosis (PBC) are rare polygenic autoimmune diseases (AIDs) characterized by fibroblast dysfunction. Furthermore, both diseases share some genetic bases with other AIDs, as evidenced by autoimmune gene pleiotropism. The present study was undertaken to investigate whether single-nucleotide polymorphisms (SNPs) identified by a large genome-wide association study (GWAS) in PBC might contribute to SSc susceptibility.
\end{abstract}

Methods: Sixteen PBC susceptibility SNPs were genotyped in a total of 1,616 patients with SSC and 3,621 healthy controls from two European populations (France and Italy).

Results: We observed an association between PLCL2 rs1372072 (odds ratio (OR) $=1.22,95 \%$ confidence interval (Cl) 1.12 to $\left.1.33, P_{\text {adj }}=7.22 \times 10^{-5}\right)$, nuclear factor-kappa-B (NF-KB) rs7665090 (OR=1.15,95\% Cl 1.06 to $\left.1.25, P_{\text {adj }}=0.01\right)$, and IRF8 rs11117432 (OR $=0.75,95 \% \mathrm{Cl} 0.67$ to $\left.0.86, P_{\text {adj }}=2.49 \times 10^{-4}\right)$ with SSc susceptibility. Furthermore, phenotype stratification showed an association between rs1372072 and rs11117432 with the limited cutaneous subgroup (IcSSc) $\left(P_{\text {adj }}=4.45 \times 10^{-4}\right.$ and $\left.P_{\text {adj }}=0.001\right)$, whereas rs 7665090 was associated with the diffuse cutaneous subtype (dcSSc) $\left(P_{\text {adj }}=0.003\right)$. Genotype-mRNA expression correlation analysis revealed that the IRF8 protective allele was associated with increased interferon-gamma (IFN- $\gamma)$ expression $(P=0.03)$ in patients with SSC but decreased type I IFN (IFIT1) expression in patients and controls $(P=0.02)$. In addition, we found an epistatic interaction between NF-KB and IRF8 $\left(\mathrm{OR}=0.56,95 \% \mathrm{Cl} 0.00\right.$ to $\left.0.74, P=4 \times 10^{-4}\right)$ which in turn revealed that the IRF8 protective effect is dependent on the presence of the NF-KB susceptibility allele.

Conclusions: An analysis of pleiotropic genes identified two new susceptibility genes for SSC (NF-KB and PLCL2) and confirmed the IRF8 locus. Furthermore, the IRF8 variant influenced the IFN signature, and we found an interaction between IRF8 and NF-KB gene variants that might play a role in SSc susceptibility.

\footnotetext{
* Correspondence: yannick.allanore@cch.aphp.fr

${ }^{1}$ Paris Descartes University, INSERM U1016, Institut Cochin, Sorbonne Paris

Cité, Paris, France

${ }^{13}$ Paris Descartes University, Rheumatology A Department, Cochin Hospital, APHP, Paris, France

Full list of author information is available at the end of the article
} 


\section{Introduction}

Systemic sclerosis (SSc) is an autoimmune disease (AID) characterized by vascular damage, autoantibody production, and fibrotic events. SSc is an orphan disease that is considered the most severe connective tissue disorder, representing an important medical challenge because of its debilitating progressive nature [1]. The precise aetiology of the disease remains unclear. However, as in other AIDs, interactions between environment and genetic factors are thought to play a key role in disease susceptibility [2]. Indeed, several reports of well-powered candidate gene studies, together with genome-wide association studies (GWASs), have established numerous SSc susceptibility genes, including STAT4, IRF5, TNFSF4, CD226, CD247, and BANK1 [3,4]. The vast majority of these susceptibility loci belong to pathways involved in immune responses or inflammation and were identified in other AIDs [3]. Previous work from our team demonstrated that polyautoimmunity affects up to a quarter of patients with SSc, highlighting the concept of shared autoimmunity in SSc [5]. It is well established that AIDs encompass a broad range of phenotypic manifestations and severity, indicating that the effects of these loci are not of equal magnitude and may not be associated in the same direction (risk or protection) among different diseases [3]. Fibroblast dysfunction, the SSc hallmark, can also be observed in primary biliary cirrhosis (PBC). PBC is a disease marked by progressive destruction of the liver and can co-occur with SSc, suggesting that these two AIDs may share common pathways and may be a result of common-variant loci of weak effect [6]. A recent GWAS carried out in patients with PBC identified 12 new loci involved in disease susceptibility and highlighted the importance of type I interferon (IFN), nuclear factor-kappa-B (NF- $\mathrm{B}$ ), and Toll-like receptor (TLR) signaling [7] in its pathogenesis. Given the evidence of shared autoimmune genes between SSc and other AIDs, we investigated the association of 16 single-nucleotide polymorphisms (SNPs), recently identified as susceptibility factors for PBC, with SSc and its subphenotypes.

\section{Methods}

\section{Study population}

We performed a case-control association study with a European Caucasian cohort consisting of 1,616 patients with SSc (1,022 French and 594 Italians) and 3,621 controls (2,384 French and 1,237 Italians). Only individuals with European ancestry (defined as all four grandparents being of European Caucasian ancestry) were included in the study. This cohort has already been used in several previous genetic studies, and high homogeneity between French and Italian samples has been demonstrated $[4,8]$. Control subjects were age- and sex-matched to patients with SSc. The characteristics of the patients with SSc are shown in Table 1. All patients with SSc were classified by LeRoy's cutaneous subtype and were phenotypically screened for anti-nuclear antibodies and pulmonary fibrosis (PF). The latter was defined as any of the following changes on computed tomography scan, such as in our previous studies: reticulations, honeycombing, and traction. In line with all of our previous projects, patients with SSc were also evaluated for the presence of other potential AIDs. To avoid any bias due to a possible excess of the risk alleles attributable to these patients, 23 SSc patients showing PBC co-occurrence were excluded from the main study. This study was approved by the Comité Consultatif de Protection des Personnes dans la Recherche Biomédicale (CCPPRB) Paris Cochin (dossier 2068). All participants enrolled in this study provided written informed consent.

Table 1 Characteristics of systemic sclerosis patients included in the study

\begin{tabular}{llll}
\hline & French cohort $(\mathbf{n = 1 , 0 2 2})$ & Italian cohort $(\mathbf{n}=\mathbf{5 9 4})$ & Combined cohort $(\mathbf{n}=\mathbf{1 , 6 1 6})$ \\
\hline Age in years, mean \pm SD & $57.4 \pm 13.9$ & $48.8 \pm 13.4$ & $53.1 \pm 13.6$ \\
Male gender, number & 152 & 55 & 207 \\
Disease duration in years, mean \pm SD & $13.1 \pm 7.1$ & $12.4 \pm 8.7$ & $12.75 \pm 7.9$ \\
ICSSC, percentage & 60 & 74.7 & 64.4 \\
dcSSC, percentage & 29.5 & 25.2 & 28 \\
Lung fibrosis seen on CT, percentage & 36.1 & 34.6 & 35.3 \\
FVC <75\%, percentage & 14 & 16.1 & 14.6 \\
Digital ulcers (ever occurred), percentage & 33 & 44 & 36.6 \\
ACA+, percentage & 32.3 & 44.6 & 36.6 \\
Anti-topo I+, percentage & 23 & 32.6 & 26.3 \\
Associated AlD, percentage & 15 & 14.6 & 15
\end{tabular}

ACA, anti-centromere antibody; anti-topo I, anti-topoisomerase antibody; associated AID, at least one associated autoimmune disease; CT, computed tomography; dcSSc, diffuse cutaneous systemic sclerosis; FVC, forced vital capacity; ICSSc, limited cutaneous systemic sclerosis; SD, standard deviation. 


\section{Single-nucleotide polymorphism selection}

The SNP selection was based on a previous PBC GWAS, which identified these SNPs as susceptibility genes to PBC [7]. Among these candidates, two SNPs, STAT4 rs10931468 and IRF5 rs12531711, located in loci reproducibly strongly associated with SSc in several studies [3,4], were excluded from the study. The list of the selected SNPs, as well as information about their localization and putative function, is shown in Additional file 1: Table S1.

\section{Genotyping}

DNA samples from SSc patients and controls were genotyped for the 16 tag SNPs: rs10752747, rs12134279, rs1372072, rs2293370, rs485499, rs7665090, rs860413, rs6974491, rs6421571, rs1800693, rs911263, rs8017161, rs11117432, rs7208487, rs3745516, and rs968451. Genotyping was performed by using a competitive allele-specific polymerase chain reaction system (KASpar genotyping; KBioscience, Hoddesdon, UK).

\section{Gene expression in peripheral blood mononuclear cells}

To assess a possible genotype-phenotype correlation, we selected the three associated genes-PLCL2, NFKB1, and $I R F 8$ - to perform gene expression assays of their mRNA. Moreover, because of compelling data on IRF8-associated pathways, we also investigated IFN- $\gamma$ and type I IFN (IFIT1) mRNA gene expression. We randomly selected peripheral blood nuclear cell (PBMC) samples from 39 patients (who did not receive immunosuppressive drugs) and 24 healthy controls. The total RNA was obtained by using an RNA extraction kit (RNeasy Mini Kit; Qiagen, Hilden, Germany) followed by a c-DNA reverse transcription step (SuperScript II Reverse Transcriptase; Invitrogen, Waltham, MA, USA). Gene expression was performed by using quantitative Real-Time Polymerase Reaction (Universal Master Mix II; Applied Biosystems, Waltham, MA, USA). All primers were obtained by a predesigned gene expression assay (Applied Biosystems).

\section{Statistical analysis}

Statistical analyses were performed by using PLINK (Harvard and MIT version 1.07, available at [9]). Power calculation was assessed by using a two independent binomial module combined with a one-sided Fisher exact test (StatXact 8 software; Cytel, Cambridge, MA, USA). Tests for conformity with Hardy-Weinberg equilibrium were performed by using a standard chi-square test (1 degree of freedom). Homogeneity between the two cohorts was confirmed by using the Breslow-Day method and therefore the combined data were subsequently analyzed by calculating the pooled odds ratios (ORs) by using a Cochran-Mantel-Haenszel test under fixed effects. Individual association analyses of all SNPs were performed by comparing cases and controls with a two-sided Fisher exact test on allelic distribution. All ORs are provided with their 95\% confidence intervals (CIs).

To test $\mathrm{SNP} \times \mathrm{SNP}$ epistasis, the allele frequencies were compared in patients and control subjects. The PLCL2*T alleles, $N F-k B^{*} \mathrm{G}$ alleles, and $I R F 8^{*} \mathrm{~A}$ alleles were considered binary random variables, and the dependency of disease on pairwise combinations of these covariates was tested with a logistic regression model. The fitted model included each one of two tested factors as first-order components and an interaction term reflecting the extent of the allelic by allelic epistasis. We applied a logistic regression assuming a double-dominant model. Exact calculations of ORs and $P$ values were made with Logxact 8 software (Cytel).

We applied the Bonferroni correction although it is very conservative; 16 tag SNPs were considered to test the association between each SNP and SSc ( $P$ values were therefore multiplied by 16 to get the adjusted ones). For disease phenotypes correction, we took into account seven subtypes ( $P$ values were multiplied by 7 to get the adjusted ones). An unpaired two-sided $t$ test was used for mRNA expression levels analysis. An adjusted $P$ value of less than 0.05 was considered statistically significant.

\section{Results}

\section{Single-marker analysis}

Sixteen SNPs were genotyped in two European populations (French and Italian). Among the SNPs of interest, minor allele frequencies (MAFs) range from $11 \%$ to $44 \%$; therefore, we provide a power calculation for these two MAFs. For an 11\% MAF, our power to detect an association is more than $99 \%$ for an OR of 1.5 and is $81 \%$ to detect an OR of 1.3. For a $44 \%$ MAF, our power is more than $99 \%$ to detect an OR of 1.3 or higher. Genotype frequencies were in Hardy-Weinberg equilibrium in the control population for all of the SNPs investigated.

We first evaluated the French sample and observed six loci for which a nominal $P$ value was reached: PLCL2 rs1372072 OR $=1.22$, 95\% CI 1.10 to $1.36, P=1.97 \times 10^{-4}$; TIMMDC1 rs2293370 OR $=0.84,95 \%$ CI 0.73 to 0.96 , $P=1.46 \times 10^{-2} ; I L 12 A$ rs 485499 OR $=0.88,95 \%$ CI 0.79 to $0.99, P=3.37 \times 10^{-2} ; N F-k B$ rs7665090 OR $=1.12,95 \% \mathrm{CI}$ 1.01 to $1.25, P=2.47 \times 10^{-2}$; IL7R rs860413 OR=0.89, $95 \%$ CI 0.79 to $1.00, P=5.77 \times 10^{-2}$; and IRF8 rs11117432 $\mathrm{OR}=0.68,95 \%$ CI 0.68 to $0.93, P=6.52 \times 10^{-3}$. We then analysed all the SNPs in the Italian sample and observed that five SNPs, including three previously identified in the French sample, were associated with SSc: PLCL2 rs1372072 OR $=1.22,95 \%$ CI 1.05 to $1.41, P=7.76 \times 10^{-3}$; $N F-k B$ rs7665090 OR $=1.20,95 \%$ CI 1.04 to 1.38 , $P=9.00 \times 10^{-3} ; E L M O 1 \mathrm{rs} 6974491 \mathrm{OR}=1.27,95 \%$ CI 1.04 to $1.55, P=1.93 \times 10^{-2} ; R A D 51 B$ rs911263 OR $=0.80,95 \%$ CI 0.69 to $0.93, P=4.18 \times 10^{-3}$; and IRF8 rs 11117432 OR $=0.70,95 \%$ CI 0.57 to $0.85, P=5.48 \times 19^{-4}$. It is of 
note that, when Bonferroni correction was applied in each population, only PLCL2 rs1372072 $P=3 \times 10^{-3}$ and IRF8 rs11117432 OR $=0.68,95 \%$ CI 0.68 to $0.93, P=8.76 \times 10^{-3}$ remained significant in French and Italian samples, respectively (Table 2).

Therefore, to increase the statistical power, we moved for combined analyses taking into account that homogeneity of the cohorts was confirmed by the Breslow-Day test (no evidence of interpopulation heterogeneity) (Table 3). Our combined analysis revealed a significant association for three of the studied SNPs: PLCL2 rs1372072 OR $=1.22$, 95\% CI 1.12 to $1.33, P_{\text {adj }}=7.22 \times 10^{-5} ; N F-k B$ rs7665090 OR $=1.15$, 95\% CI 1.06 to $1.25, P_{\text {adj }}=0.01$; and IRF8 rs11117432, OR $=0.75,95 \%$ CI 0.67 to $0.86, P_{\text {adj }}=2.49 \times 10^{-4}$ (Table 3). It is noteworthy that combined analyses revealed the same directions of effect for the three SNPs and, of the most importance, that the combined $P$ value was greater than the $P$ value observed in a single population, altogether, this supports a true association.

The next step was to investigate possible associations between $P L C L 2, I R F 8$, and $N F-\kappa B$ polymorphisms with clinical features. For that purpose, we stratified the patients according to their main subphenotypes in the combined sample. After Bonferroni correction for multiple testing, we found that the variants PLCL2 rs1372072 and IRF8 rs11117432 were associated with the limited cutaneous subgroup (lcSSc) $(\mathrm{OR}=1.23,95 \% \mathrm{CI}$ 1.11 to $1.36, P_{\text {adj }}=4.45 \times 10^{-4}$ and $\mathrm{OR}=0.75,95 \%$ CI 0.65 to $0.87, P_{\text {adj }}=0.001$, respectively) and with the presence of anti-centromere antibodies (ACAs) $(\mathrm{OR}=1.29,95 \% \mathrm{CI}$ 1.13 to $1.46, P_{\text {adj }}=4.35 \times 10^{-4}$ and $\mathrm{OR}=0.78,95 \%$ CI 0.65 to $0.93, P_{\text {adj }}=0.04$ ) (Table 3).

The $N F-\kappa B$ variant rs7665090 was shown to be associated with patients belonging to a diffuse cutaneous phenotype (dcSSc) $\left(\mathrm{OR}=1.28,95 \%\right.$ CI 1.11 to $\left.1.47, P_{\text {adj }}=0.003\right)$ and also with the PF subset $(\mathrm{OR}=1.24,95 \%$ CI 1.09 to $\left.1.41, P_{\text {adj }}=0.004\right)$ (Table 3). No association was found between the DENND1B, TIMMDC1, IL7R, ELMO1, DDX6, TNFRSF1A, RAD51B, TNFAIP2, SPIB, and SNORD43 gene variants and SSc (Additional file 2: Table S2).

When the 23 SSc-PBC patients were analysed separately, no significant association was observed when they were compared with the 3,621 controls for any SNPs; furthermore, their addition to the whole sample (23 SSc-PBC $+1,628$ SSc patients) did not modify the results (significant association for PLCL2, IRF8, and NF- $K B$, with unchanged magnitude of the effects). In regard to the group of SSc patients having another AID $(\mathrm{n}=224)$, single-marker analyses revealed significant association with PLCL2 rs1372072 only $(P=0.001 ; \mathrm{OR}=1.375,95 \% \mathrm{CI} 1.13$ to 1.67$)$.

\section{Follow-up of the raised hypotheses for single markers} SNPs belonging to the genes highlighted herein have been previously reported as associated or with a trend for association in previous works. For some genes highlighted in our study, some variants not primarily herein studied have been reported as associated with SSc or as having a trend for association with SSc. Indeed, Martin et al. [10] reported a suggestive association of $N F-\kappa B$ rs1598859, and a robust association has been reported for IRF8 rs11642873 [11]. Furthermore, a recent work did suggest some association in two loci for which we herein identified a nominal association but not reaching significance after multi-test correction as we observe for DDX6 rs7130875 and IL12A rs77583790 [12].

In our sample, we therefore investigated these markers and compared the association with the one observed with our newly identified SNPs. To that end, we used a subset of our patients for whom we have performed genome-wide typing in the past (sample of 564 patients with SSc and 488 healthy controls) [13]. In regard to NF- $\mathrm{kB}$, we first observed in HapMap that rs1598859 and rs7665090 are not in complete linkage disequilibrium (LD) $\quad\left(\mathrm{D}^{\prime}=0.77\right.$ and $\mathrm{R}^{2}=0.31$; SNPs are on the same block according to $\mathrm{D}^{\prime}$ value but are not in phase according to $\mathrm{R}^{2}$ ) and then found from our GWAS data that the MAFs were 0.355 in patients with SSc and 0.382 in controls (uncorrected $P$ value $=0.2$ ). For IRF8, an established locus, we first observed that rs11642873 and rs11117432 are not in complete LD $\left(D^{\prime}=0.707\right.$ and $\left.R^{2}=0.456\right)$. Within our subset with GWAS data, we identified that the rs 11642873 MAFs were 0.14 in the patients with SSc and 0.174 in the controls $(P$ value $=0.034$ without correction; $\mathrm{OR}=0.772,95 \% \mathrm{CI}$ 0.61 to 0.98$)$. The $P$ value is less significant than the one obtained for rs11117432 in the same subset $(\mathrm{MAF}=0.156$ versus $0.213 ; P=0.0008$ ), and the $\mathrm{OR}$ also shows a weaker effect (OR $=0.68,95 \% \mathrm{CI} 0.55$ to 0.85 ). We also performed haplotype analyses to estimate the effects of the two SNPs and that relates to conditional analyses; we observed that the haplotype combination including the single rs11642873 risk variant (CG haplotype) was not associated with SSc risk (frequency of 0.108 versus $0.117 ; P=0.499$ ) but that the haplotype combination including the single rs11117432 risk variant (AA haplotype) was significantly associated with SSc risk (0.124 versus 0.156; $P=0.032)$. We proceeded similarly for $D D X 6$ rs7130875 and observed that it was not in LD with rs6421571 $\left(\mathrm{D}^{\prime}=0.839\right.$ and $\left.\mathrm{R}^{2}=0.084\right)$. DDX6 rs7130875 was not included in our array, but we used the perfect proxy rs4499035 and identified that the MAFs were 0.261 in patients with SSc and 0.252 in the controls (uncorrected $P$ value $=0.69$ ). IL12A rs77583790 SNP was not included in the array used in our GWAS, and no accurate imputation could be done. Therefore, we have performed a dedicated genotyping by using the TaqMan method in a subset of the patients and controls for whom DNA was still available and who did participate in the present study; we found that rs77583790 minor A allele was present in $2.33 \%$ of 1,585 patients with SSc and in 
Table 2 Analysis of PLCL2 rs1372072, NF-KB rs7665090, and IRF8 rs11117432 gene variants in French and Italian populations

\begin{tabular}{|c|c|c|c|c|c|c|c|}
\hline \multirow{2}{*}{$\begin{array}{l}\text { SNP, phenotype (n) } \\
\text { French Caucasian }\end{array}$} & \multirow[t]{2}{*}{ MAF } & \multicolumn{3}{|c|}{ Genotype distribution } & \multirow[t]{2}{*}{$P$ value } & \multirow[t]{2}{*}{$P_{\text {adj }}^{\mathrm{a}}$} & \multirow[t]{2}{*}{ OR $(95 \% \mathrm{Cl})$} \\
\hline & & & & & & & \\
\hline PLCL2 rs1372072 & T & Tा (\%) & TC (\%) & CC (\%) & & & \\
\hline $\operatorname{SSC}(1,021)$ & 0.39 & 15.40 & 47.41 & 37.17 & $1.97 \times 10^{-4}$ & $3.15 \times 10^{-3}$ & $1.22(1.10-1.36)$ \\
\hline dcSSc (298) & 0.39 & 15.64 & 46.93 & 37.41 & 0.02 & 0.39 & $1.22(1.03-1.46)$ \\
\hline SSc. Topo I+ (233) & 0.39 & 15.51 & 48.27 & 36.20 & 0.02 & 0.39 & $1.25(1.03-1.52)$ \\
\hline ICSSC (591) & 0.38 & 15.29 & 47.07 & 37.62 & $4.45 \times 10^{-3}$ & 0.07 & $1.21(1.06-1.38)$ \\
\hline SSC. ACA+ (349) & 0.39 & 16.90 & 45.77 & 37.31 & $5.37 \times 10^{-3}$ & 0.08 & $1.26(1.07-1.49)$ \\
\hline Pulmonary fibrosis (364) & 0.40 & 16.62 & 48.47 & 34.90 & $6.97 \times 10^{-4}$ & 0.01 & $1.32(1.12-1.55)$ \\
\hline Controls $(2,384)$ & 0.34 & 12.31 & 44.01 & 43.67 & NA & NA & NA \\
\hline NF-KB rs7665090 & G & GG (\%) & AG (\%) & AA (\%) & & & \\
\hline SSC $(1,021)$ & 0.52 & 27.21 & 49.59 & 23.18 & 0.02 & 0.39 & $1.12(1.01-1.25)$ \\
\hline dcSSc (298) & 0.54 & 32.29 & 44.79 & 22.91 & 0.01 & 0.16 & $1.25(1.05-1.49)$ \\
\hline SSc. Topo I+ (233) & 0.54 & 31.14 & 47.36 & 21.49 & 0.01 & 0.29 & $1.26(1.04-1.53)$ \\
\hline ICSSC (591) & 0.50 & 24.95 & 51.81 & 23.22 & 0.26 & NS & $1.07(0.94-1.22)$ \\
\hline SSC. ACA+ (349) & 0.50 & 23.97 & 52.04 & 23.97 & 0.65 & NS & $1.04(0.88-1.22)$ \\
\hline Pulmonary fibrosis (364) & 0.52 & 28.53 & 48.87 & 22.59 & 0.05 & 0.84 & $1.17(1.00-1.37)$ \\
\hline Controls $(2,384)$ & 0.49 & 23.35 & 51.31 & 25.33 & NA & NA & NA \\
\hline IRF8 rs11117432 & A & AA (\%) & AG (\%) & GG (\%) & & & \\
\hline $\operatorname{SSC}(1,021)$ & 0.16 & 2.53 & 27.28 & 70.18 & $6.52 \times 10^{-3}$ & 0.10 & $0.79(0.68-0.93)$ \\
\hline dcSSc (298) & 0.15 & 2.38 & 25.25 & 72.35 & 0.01 & 0.22 & $0.73(0.56-0.94)$ \\
\hline SSc. Topo I+ (233) & 0.16 & 3.52 & 26.87 & 69.60 & 0.23 & NS & $0.84(0.64-1.10)$ \\
\hline ICSSC (591) & 0.16 & 2.28 & 29.22 & 68.48 & 0.08 & NS & $0.84(0.69-1.01)$ \\
\hline SSc. ACA+ (349) & 0.15 & 3.28 & 25.37 & 71.34 & 0.04 & 0.74 & $0.78(0.62-0.99)$ \\
\hline Pulmonary fibrosis (364) & 0.16 & 2.26 & 27.76 & 69.97 & 0.05 & 0.82 & $0.79(0.63-1.00)$ \\
\hline Controls $(2,384)$ & 0.19 & 3.77 & 31.33 & 64.88 & NA & NA & NA \\
\hline \multicolumn{8}{|l|}{ Italian Caucasian } \\
\hline PLCL2 rs1372072 & T & TТ (\%) & TC (\%) & CC (\%) & & & \\
\hline SSC (607) & 0.36 & 13.36 & 47.03 & 39.59 & $7.76 \times 10^{-3}$ & 0.12 & $1.22(1.05-1.41)$ \\
\hline dcSSc (152) & 0.34 & 11.72 & 46.20 & 42.06 & 0.42 & NS & $1.11(0.86-1.44)$ \\
\hline SSc. Topo I+ (233) & 0.35 & 11.57 & 47.36 & 41.05 & 0.29 & NS & $1.13(0.90-1.42)$ \\
\hline ICSSC (455) & 0.37 & 13.90 & 47.30 & 38.78 & $5.55 \times 10^{-3}$ & 0.08 & $1.25(1.06-1.47)$ \\
\hline SSC. ACA+ (349) & 0.38 & 14.66 & 48.49 & 36.84 & $4.49 \times 10^{-3}$ & 0.07 & $1.32(1.09-1.61)$ \\
\hline Pulmonary fibrosis (210) & 0.39 & 13.72 & 50.98 & 35.29 & $7.62 \times 10^{-3}$ & 0.12 & $1.34(1.08-1.67)$ \\
\hline Controls $(1,237)$ & 0.32 & 10.68 & 43.41 & 45.89 & NA & NA & NA \\
\hline NF-KB rs7665090 & G & GG (\%) & AG (\%) & AA (\%) & & & \\
\hline SSc (607) & 0.52 & 29.66 & 47.45 & 24.23 & $9.00 \times 10^{-3}$ & 0.14 & $1.20(1.08-1.33)$ \\
\hline dcSSc (152) & 0.55 & 33.33 & 44.02 & 22.66 & 0.01 & 0.31 & $1.34(1.03-1.76)$ \\
\hline SSc. Topo I+ (233) & 0.50 & 24.61 & 51.79 & 23.58 & 0.38 & NS & $1.10(0.81-1.37)$ \\
\hline ICSSC (455) & 0.51 & 27.90 & 47.76 & 24.33 & 0.05 & 0.89 & $1.16(0.96-1.34)$ \\
\hline SSC. ACA+ (349) & 0.51 & 27.50 & 47.58 & 24.90 & 0.18 & NS & $1.14(0.95-1.34)$ \\
\hline Pulmonary fibrosis (210) & 0.56 & 34.61 & 42.78 & 22.59 & $2.29 \times 10^{-3}$ & 0.04 & $1.37(1.17-1.68)$ \\
\hline Controls $(1,237)$ & 0.48 & 22.60 & 50.85 & 26.53 & NA & NA & NA \\
\hline IRF8 rs 11117432 & A & AA (\%) & AG (\%) & GG (\%) & & & \\
\hline
\end{tabular}


Table 2 Analysis of PLCL2 rs1372072, NF-KB rs7665090, and IRF8 rs11117432 gene variants in French and Italian populations (Continued)

\begin{tabular}{|c|c|c|c|c|c|c|c|}
\hline SSC (607) & 0.12 & 1.17 & 23.23 & 75.58 & $5.47 \times 10^{-4}$ & $8.76 \times 10^{-3}$ & $0.70(0.57-0.85)$ \\
\hline dcSSc (152) & 0.14 & 2.64 & 24.50 & 72.84 & 0.33 & NS & $0.83(0.60-1.17)$ \\
\hline SSc. Topo I+ (233) & 0.12 & 2.04 & 21.42 & 76.53 & 0.02 & 0.44 & $0.70(0.51-0.96)$ \\
\hline IcSSc (455) & 0.12 & 0.67 & 22.79 & 76.52 & $2.51 \times 10^{-4}$ & $4 \times 10^{-3}$ & $0.65(0.52-0.82)$ \\
\hline SSC. ACA+ (349) & 0.13 & 1.14 & 25.47 & 73.38 & 0.06 & 0.97 & $0.77(0.59-1.01)$ \\
\hline Pulmonary fibrosis (210) & 0.12 & 1.92 & 22.11 & 75.96 & 0.03 & 0.51 & $0.71(0.52-0.96)$ \\
\hline Controls $(1,237)$ & 0.17 & 3.15 & 28.21 & 68.63 & NA & NA & NA \\
\hline
\end{tabular}

${ }^{a}$ After Bonferroni correction. ACA+, anti-centromere antibody; $\mathrm{Cl}$, confidence interval; dcSSc, diffuse cutaneous systemic sclerosis; IcSSc, limited cutaneous systemic sclerosis; MAF, minor allele frequency; n, number of pooled patients analysed; NA, not applicable; NS, not significant; OR, odds ratio; SNP, single-nucleotide polymorphism; SSc, systemic sclerosis; Topo I+, anti-topoisomerase I antibody.

$1.36 \%$ of 1,842 healthy controls showing a nominal association but not supporting any correction for multiple testing (uncorrected $P=0.04$ ). We have thereafter performed some meta-analysis by using the data included in the cited works [10-12] together with ours, thanks to Pr Javier Martin, who provided the genotypic data from his original studies (the sample size was not always the same as in the original study which could include several cohorts from various origins). As shown in Additional file 3: Table S3, neither the $P$ value nor the OR was improved for NF- $K B, D D X 6$, and IL12A genes. This suggests that adding our data does not strengthen the previous findings and does not support a

Table 3 Analysis of PLCL2 rs1372072, NF-KB rs7665090, and IRF8 rs11117432 gene variants in the combined Caucasian populations (French and Italian)

\begin{tabular}{|c|c|c|c|c|c|c|c|}
\hline \multirow{2}{*}{$\frac{\text { SNP, phenotype (n) }}{P L C L 2 \text { rs } 1372072}$} & \multirow{2}{*}{$\begin{array}{l}\text { MAF } \\
T\end{array}$} & \multicolumn{3}{|c|}{ Genotype distribution } & \multirow[t]{2}{*}{$P$ value } & \multirow[t]{2}{*}{$P_{\text {adj }}^{\mathrm{a}}$} & \multirow[t]{2}{*}{ OR $(95 \% \mathrm{Cl})$} \\
\hline & & $\Pi(\%)$ & TC (\%) & CC (\%) & & & \\
\hline SSC $(1,597)$ & 0.38 & 14.65 & 47.27 & 38.07 & $4.01 \times 10^{-6}$ & $7.22 \times 10^{-5}$ & $1.22(1.12-1.33)$ \\
\hline dcSSc (439) & 0.38 & 14.35 & 46.69 & 38.95 & 0.01 & 0.12 & $1.19(1.03-1.37)$ \\
\hline SSc, Topo I+ (422) & 0.38 & 13.74 & 47.86 & 38.38 & 0.01 & 0.09 & $1.20(1.03-1.39)$ \\
\hline IcSSc $(1,028)$ & 0.38 & 14.68 & 47.17 & 38.13 & $6.37 \times 10^{-5}$ & $4.45 \times 10^{-4}$ & $1.23(1.11-1.36)$ \\
\hline SSC, ACA+ (609) & 0.39 & 15.92 & 46.96 & 37.11 & $6.21 \times 10^{-5}$ & $4.35 \times 10^{-4}$ & $1.29(1.13-1.46)$ \\
\hline Pulmonary fibrosis (565) & 0.40 & 15.57 & 49.38 & 35.04 & $1.29 \times 10^{-5}$ & $9.07 \times 10^{-5}$ & $1.33(1.17-1.51)$ \\
\hline Controls $(3,570)$ & 0.33 & 11.76 & 43.80 & 44.42 & NA & NA & NA \\
\hline NF-KB rs7665090 & G & GG (\%) & AG (\%) & AA (\%) & & & \\
\hline $\operatorname{SSc}(1,590)$ & 0.52 & 27.98 & 48.55 & 23.45 & $7.19 \times 10^{-4}$ & 0.01 & $1.15(1.06-1.25)$ \\
\hline dcSSc (438) & 0.55 & 32.64 & 44.52 & 22.83 & $4.93 \times 10^{-4}$ & 0.003 & $1.28(1.11-1.47)$ \\
\hline SSc, Topo I+ (423) & 0.53 & 28.13 & 49.40 & 22.45 & 0.01 & 0.12 & $1.18(1.03-1.37)$ \\
\hline ICSSC $(1,025)$ & 0.51 & 26.24 & 50.04 & 23.70 & 0.03 & 0.24 & $1.11(1.00-1.22)$ \\
\hline $\mathrm{SSC}, \mathrm{ACA}+(611)$ & 0.51 & 25.53 & 50.08 & 24.38 & 0.20 & NS & $1.08(0.95-1.22)$ \\
\hline Pulmonary fibrosis (562) & 0.54 & 30.78 & 46.61 & 22.59 & $7.14 \times 10^{-4}$ & 0.004 & $1.24(1.09-1.41)$ \\
\hline Controls $(3,585)$ & 0.48 & 23.09 & 51.15 & 25.74 & NA & NA & NA \\
\hline IRF8 rs11117432 & A & AA (\%) & AG (\%) & GG (\%) & & & \\
\hline $\operatorname{SSC}(1,500)$ & 0.14 & 2.03 & 25.76 & 72.22 & $1.56 \times 10^{-5}$ & $2.49 \times 10^{-4}$ & $0.75(0.67-0.86)$ \\
\hline dcSSc (444) & 0.15 & 2.47 & 25 & 72.52 & 0.009 & 0.06 & $0.76(0.62-0.93)$ \\
\hline SSc, Topo I+ (423) & 0.15 & 2.83 & 24.34 & 72.81 & 0.01 & 0.11 & $0.77(0.63-0.95)$ \\
\hline ICSSC $(1,011)$ & 0.14 & 1.58 & 26.40 & 72.00 & $1.96 \times 10^{-4}$ & 0.001 & $0.75(0.65-0.87)$ \\
\hline $\mathrm{SSC}, \mathrm{ACA}+(598)$ & 0.15 & 2.34 & 25.41 & 72.24 & 0.005 & 0.04 & $0.78(0.65-0.93)$ \\
\hline Pulmonary fibrosis (561) & 0.15 & 2.13 & 25.66 & 72.19 & 0.004 & 0.02 & $0.76(0.63-0.91)$ \\
\hline Controls $(2,290)$ & 0.18 & 3.44 & 29.69 & 66.85 & NA & NA & NA \\
\hline
\end{tabular}

${ }^{a}$ After Bonferroni correction. ACA+, anti-centromere antibody; $\mathrm{Cl}$, confidence interval; dcSSc, diffuse cutaneous systemic sclerosis; IcSSc, limited cutaneous systemic sclerosis; MAF, minor allele frequency; n, number of pooled patients analysed; NA, not applicable; NS, not significant; OR, odds ratio; SNP, single-nucleotide polymorphism; SSC, systemic sclerosis; Topo I+, anti-topoisomerase I antibody. 
significant association for $N F-\kappa B \quad r s 1598859$ and $D D X 6$ rs7130875. In regard to IL12A rs77583790, our results do not provide an independent replication; however, the meta-analysis shows a more significant $P$ value when the French population is added, suggesting that this locus is further confirmed by our data. For IRF8 rs11642873, our data decrease the $P$ value, and although the $\mathrm{OR}$ is unchanged, the $\mathrm{CI}$ is reduced, suggesting that the association is both stronger and of higher magnitude (Additional file 3: Table S3).

\section{Joint effects of $P L C L 2, N F-K B$, and IRF8 risk alleles on systemic sclerosis susceptibility}

We then investigated the joint effects of PLCL2 rs1372072*T, NF- $K B$ rs7665090*G, and IRF8 rs1117432*A risk alleles on SSc susceptibility. The ORs for SSc were 0.74 ( $95 \%$ CI 0.62 to 0.90 ) for carriers of one or two risk alleles, 0.94 ( $95 \% \mathrm{CI} 0.79$ to 1.11$)$ for carriers of three risk alleles, 0.88 (95\% CI 0.77 to 1.01) for carriers of four alleles, 1.19 (95\% CI 1.03 to 1.38) for carriers of five alleles, and 1.70 (95\% CI 1.32 to 2.19) for carriers of six risk alleles. Figure 1 shows the ORs for SSc patients with one or two and three to six risk alleles. Our results demonstrate that the risk of SSc increases proportionally as the number of alleles increases, with an OR of 1.70 when six risk alleles are considered (Figure 1).

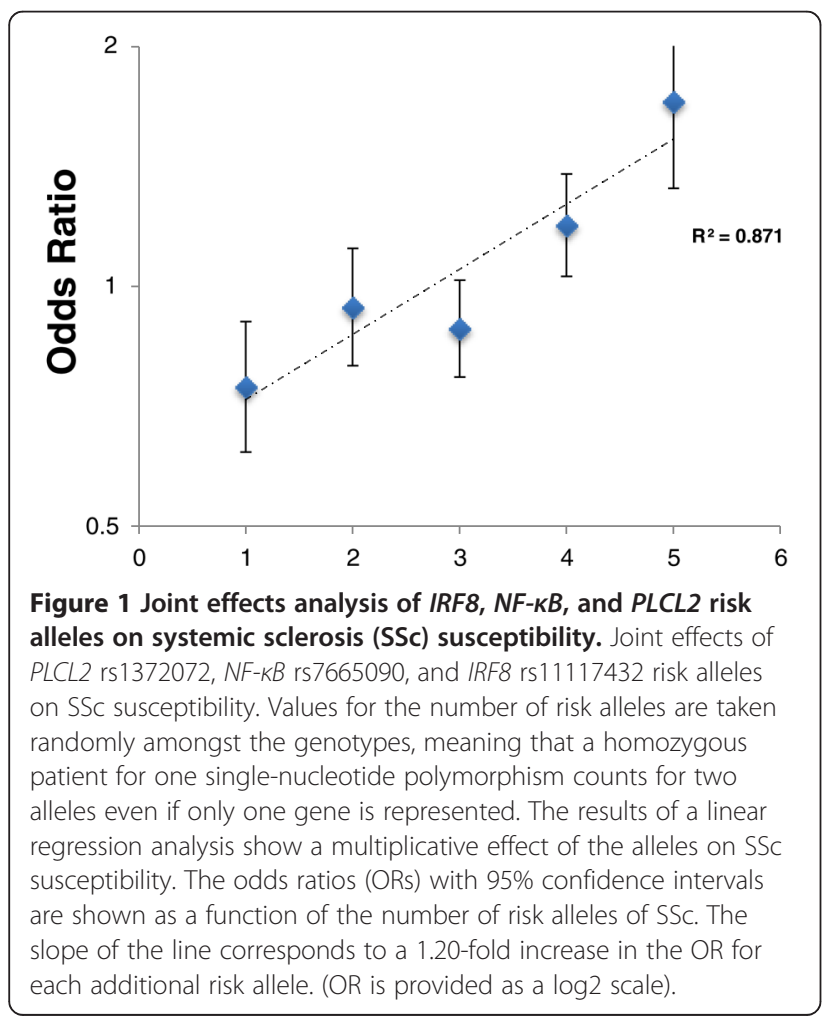

Genetic interaction between associated single-nucleotide polymorphisms

To investigate possible gene-gene interactions between the associated SNPs, we applied a logistic regression (multivariate analysis) on PLCL2 rs1372072*T, NF-KB rs7665090*G, and IRF8 rs1117432*A alleles. Of the most interest, we found an epistatic interaction between IRF8 and $N F-\kappa B\left(\mathrm{OR}=0.56,95 \%\right.$ CI 0.00 to $\left.0.74, P=4 \times 10^{-4}\right)$, which in turn demonstrated that the protective effect of the IRF8 A allele is revealed when the $N F-\kappa B$ G allele is present (Figure 2A and $\mathrm{B}$ ). Indeed, when patients were stratified according to the presence of susceptibility alleles $\mathrm{A}$ and $\mathrm{G}$, we confirmed that the IRF8 A protective effect is dependent on the presence of the $N F-\kappa B$ G allele $(N F-\kappa B$ GG or AG: $\mathrm{OR}=0.66,95 \%$ CI 0.57 to $0.77, P=5.91 \times 10^{-8}$ and $N F-\kappa B$ AA: $\mathrm{OR}=1.10,95 \%$ CI 0.85 to $1.41, P=0.45$ ). Consistent with that, $N F-\kappa B$ risk OR was observed only in patients without the protective allele of IRF8 A (IRF8 GG: $\mathrm{OR}=1.23,95 \%$ CI 1.10 to $1.38, P=1 \times 10^{-4}$ and IRF8 AA or AG: $\mathrm{OR}=0.93,95 \%$ CI 0.79 to $1.11, P=0.46$ ) (Figure 2C).

\section{mRNA expression}

We then investigated the potential influence of the three associated gene variants PLCL2, NF- $K B$, and IRF8 on their mRNA expression levels in 38 patients with SSc and 24 controls. After stratification according to the various genotypes, we observed no genotype-phenotype correlation. However, we further analyzed a possible effect of the variant IRF8 rs11117432 on IFIT1 and IFN- $\gamma$ mRNA relative expression in the same 38 patients with SSc (nine of them with the IRF8 rs11117432 AA or GA genotypes and 29 with the GG genotype) and 24 controls (12 with the IRF8 rs11117432 AA or GA genotypes and 12 with the GG genotype) according to the published data on IRF8 effects on IFN signature. Using a multiple linear regression test, we found out that the IRF8 rs $11117432 \% \mathrm{~A}$ allele was associated with IFIT1 mRNA expression levels independently of the status of the included individuals $(P=0.022$, data not shown). When all individuals were considered, our results demonstrated that the IRF8 $\mathrm{rs} 11117432^{*}$ A variant was associated with decreased levels of IFIT1 mRNA expression $(P=0.02)$. When patients and controls were considered separately, a trend was observed in the comparison of the IRF8 rs11117432*A carriers with the non-carriers $(P=0.10$ for patients, $P=0.06)$ (Figure $3 \mathrm{~A})$. We also observed increased levels of IFN $-\gamma$ mRNA expression in SSc patient carriers of $I R F 8$ rs $11117432 *$ A when compared with the non-carriers $(P=0.03)$ with a trend for difference for the whole group $(P=0.11)$ but no difference in controls $(P=0.35)$ (Figure $3 \mathrm{~B})$. There was no effect of the epistatic interaction on IFIT1 or IFN- $\gamma$ expression $(P=0.59$ for IFIT1 and $P=0.50$ for IFN- $\gamma)$. 


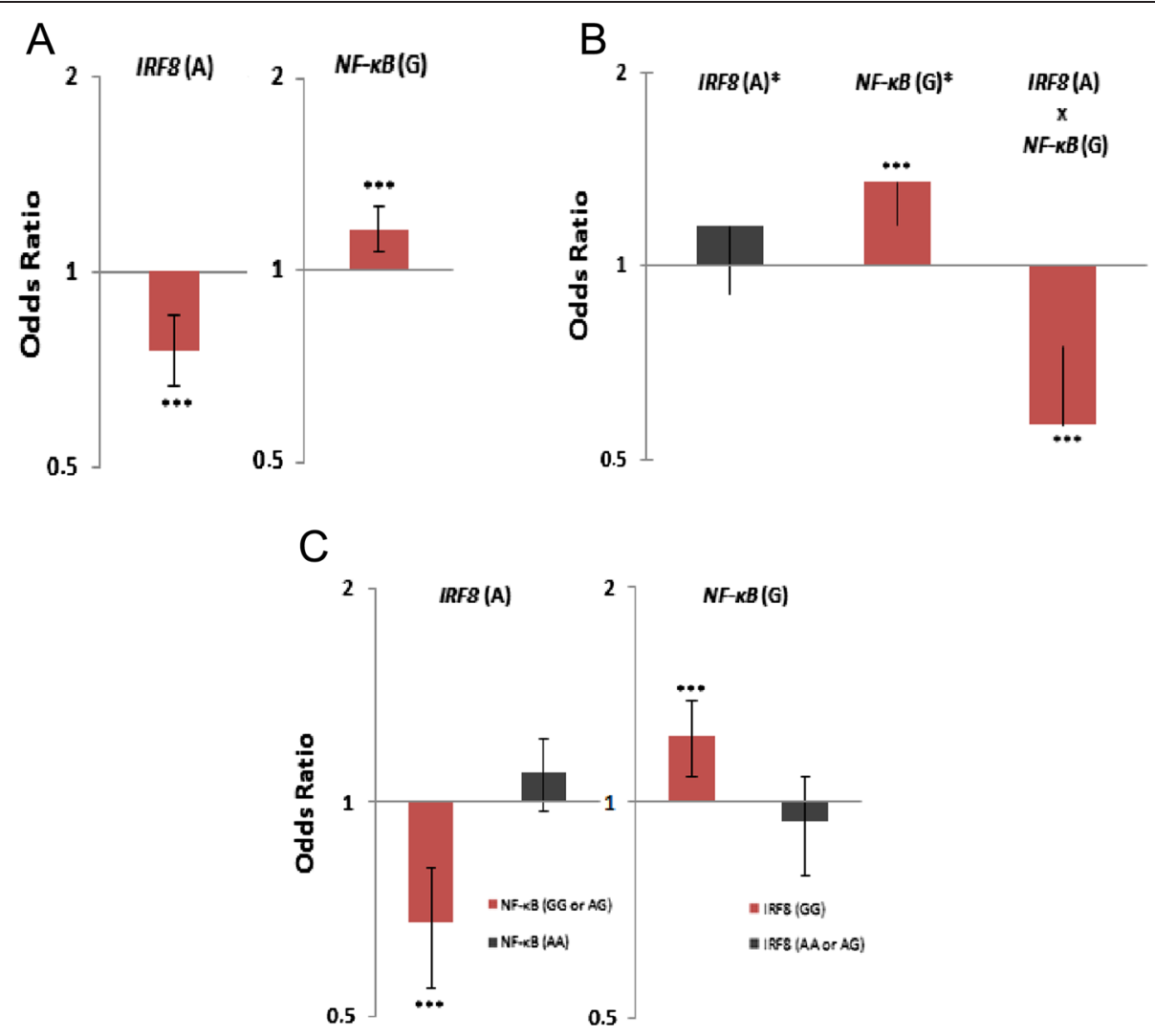

Figure 2 Analysis of the interaction between IRF8 (A) and NF-KB (G) alleles. (A) Odds ratios (ORs) and 95\% confidence intervals attributed to IRF8 rs $11117432 \mathrm{~A}$ and NF-KB rs7665090 G single-nucleotide polymorphisms (SNPs) after systemic sclerosis (SSC) association analysis. (B) Individual effect of IRF8 A and NF-KB G compared with the combined effect of both SNPs after logistic regression analysis, demonstrating that the protective effect of IRF8 A is observed only when NF-KB G is present. The black column represents the group of patients carrying IRF8 (A) allele who did not carry NF-kB (G) allele, indicating a risk effect for IRF8 (A) (OR=1.15). The grey column represents patients only harboring NF- $k B(G)$ allele $(O R=1.35)$. The red column represents the group of patients who carried IRF8 (A) and NF-KB (G) at the same time, indicating that the protective effect of IRF8 (A) is observed only when NF-KB (G) is present $(\mathrm{OR}=0.56)$. (C) Analysis of the effect of the susceptible alleles in patients stratified by the presence of NF-KB G or IRF8 A. (OR is provided as a $\log 2$ scale.) ${ }^{* * *} P<0.05$.

\section{Discussion}

The present study identified $N F-K B$ and PLCL2 as new SSc susceptibility genes and confirmed the IRF8 locus. We also showed potential effects on the IFN signature in regard to the IRF8 gene variant and an interaction between IRF8 and NF- $k B$ genes.

Our results corroborate previously reported associations in regard to the IRF8 gene and SSc susceptibility. Here, we demonstrate, for the first time, the specific association between IRF8 rs11117432 SNP and SSc susceptibility and provide data suggesting a stronger association for rs11117432 as compared with rs11642873, although our meta-analysis results strengthen the previous findings for this marker [11]. Indeed, our haplotype analyses further confirm a stronger association for rs 11117432 for a subset of our patients, but a denser genotyping would be required to better narrow a potential causal variant. IRF8 is a nuclear protein, which upon stimulation by pathogenassociated molecular pattern molecules (PAMPs) moves into the cytoplasm and activates NF- $\mathrm{kB}$ and TLR signaling pathways, leading to cytokine production and thereby regulating inflammatory responses [14]. The consequences of unregulated inflammation are associated with the development of pathologic fibrosis such as that seen in SSc [15]. Here, we show that the variant IRF8 rs11117432 increases IFN- $\gamma$ but decreases IFIT1 gene expression. Despite reaching significance, our results show subtle differences, and given the complex regulation of protein synthesis and activity, more work is required to confirm the functional impacts of the IRF 8 variant. IFNs are a group of cytokines that play an important role in the regulation of inflammation [16]. Type I IFNs, such as IFIT1, seem to contribute to the upregulation of TLR-induced inflammation and transforming growth factor-beta (TGF- $\beta$ )-responsive genes expressed by fibroblasts, whereas IFN- $\gamma$ is a potent suppressor of the pro-inflammatory TGF- $\beta$ signaling pathway, which in turn reduces the production of collagen I synthesis critical to sustain profibrotic processes in the 


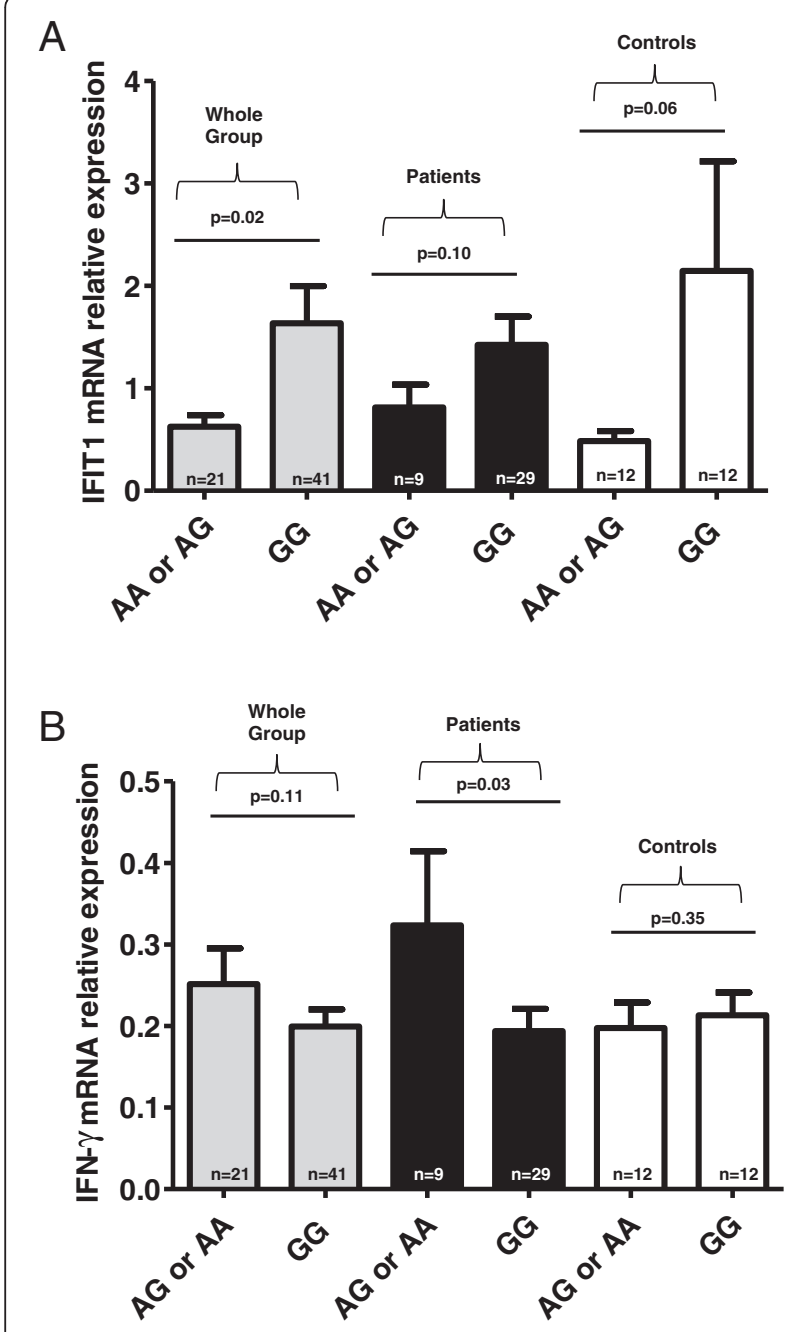

Figure 3 Type I interferon (IFIT1) and interferon-gamma (IFN- $\gamma$ ) mRNA expression levels in patients with systemic sclerosis (SSc) and healthy controls. (A) Influence of IRF8 genotype on IFIT1 mRNA expression in peripheral blood nuclear cells (PBMCs) from patients with SSC and healthy controls (mean \pm standard deviation values; uncorrected $P$ values). Expression of IFIT1 mRNA was decreased in carriers of the rs11117432 A allele as compared with non-carriers of the A allele. Vertical bars indicate the means. (B) Influence of IRF8 genotype on IFN- $\gamma$ mRNA expression in PBMCs from patients with SSc and healthy controls. Expression of IFN- $\gamma$ mRNA was increased in SSc patient carriers of the rs 11117432 A allele as compared with non-carriers of the A allele. Vertical bars indicate the means.

extracellular matrix [17-19]. Therefore, our results are in agreement with the role played by those specific cytokines and support the protective role of the IRF8 rs 11117432 variant. Thus, $I R F 8$ polymorphisms might have an effect on pro- and anti-inflammatory modulation, along with regulation of the extracellular matrix, and collagen deposition in fibrotic diseases. A recent study of serologic and cytokine profiles in patients with systemic lupus erythematosus also demonstrated an association between an IRF8 variant (rs17445836) and decreased levels of IFIT1 [20]. Moreover, according to HapMap data, the SNPs rs11117432 and rs17445836 are located in a regulatory region, underlying potential functional effects of those SNPs. In SSc, IRF8 SNPs rs11642873 and rs2280381 were already reported as being associated with disease susceptibility. It must be pointed out that rs11117432 and rs2280381 belong to the same block of LD, suggesting a strong association between the IRF8 region and SSc [11,21]. Of interest, we also identified that the association was preferentially observed in the lcSSc subset and its usual associated anti-nuclear auto-antibody (anti-centromere), suggesting a potential role for the pathogenesis in this subgroup of patients. Epidemiological studies indicate that lcSSc and anticentromere-positive patients are often associated with other AIDs, including secondary Sjögren thyroiditis and PBC, where IFIT1 is known to play a critical role [5,6].

As a complex polygenic AID, SSc is thought to have innumerous genes play a role in its aetiology and heterogeneity. However, only a handful of genes have been identified as SSc risk variants. Moreover, these variants confer relatively small increments in risk and might explain only a small proportion of the trait heritability, suggesting that other loci remain to be discovered. Here, we describe for the first time the association between $N F-\kappa B$ rs7665090 and SSc susceptibility. A previous work suggested that $N F-\kappa B$ rs1598859 had a suggestive association with SSc [10]; our results do support such an association after the analyses of a subset of our sample and by the performance of a meta-analysis combining our results together with the previously reported ones [10]. NF- $\mathrm{KB}$ is present in the cytoplasm of virtually all cell types in an active form associated with the inhibitory $\kappa \mathrm{B}(\mathrm{I} \mathrm{\kappa B})$ and has a key role in primarily immune responses related to antigen recognition, being commonly associated with the development of autoimmune and chronic inflammatory diseases [22]. Of interest, the dysregulation of NF- $\mathrm{KB}$ activity has already been implicated in the pathogenesis of systemic lupus erythematosus [23]. In SSc, the functional implications of $N F-\kappa B$ polymorphisms are still unclear. The analysis of $N F-\kappa B$ rs 7665090 variant demonstrated no effect on the levels of NF- $\mathrm{KB}$ mRNA expression; this is a false-negative result, which may have been due to the small sample size of PBMCs obtained from individuals with SSc. Further studies with a larger sample size might better elucidate the influence of $N F-K B$ rs7665090 variant in gene expression patterns. It is worth mentioning that, in a previous work, our group identified an association between TNIP1 and TNFAIP3 with SSc, genes that are known negative regulators of the NF- $\mathrm{KB}$ signaling pathway, suggesting that $N F-\kappa B$ disturbances might have a role in SSc susceptibility [13].

Our single-marker analyses also revealed an association of PLCL2 rs1372072 (OR=1.23, 95\% CI 1.12 to 1.33 , $P_{\text {adj }}=7.22 \times 10^{-5}$ ) with SSc. Phospholipase C (PLC) 
proteins are important mediators of the calcium-protein kinase $\mathrm{C}$ signaling pathway known to be expressed in hematopoietic cells, which play a role in B-cell receptor signaling and other immune responses [24]. The PLCL2 rs 4535211 variant has been reported in RA and psoriatic arthritis. The variant was associated with RA risk, whereas in psoriatic arthritis it was found to be protective [25,26]. According to HapMap data, rs1372072 and rs4535211 are in high LD $\left(\mathrm{D}^{\prime}=0.90\right)$ in a block that contains the PLCL2 gene although not in phase $\left(R^{2}=0.44\right)$. Therefore, our findings imply that this locus might contribute to several AIDs; this is an observation that must be confirmed by denser mapping and additional functional experiments to further increase our understanding of the role of PLCL2 in AIDs and SSc.

We followed up two other genes (DDX6 and IL12A) for which signals of association with SSc were recently described by another study [12] and because we observed for these 2 genes a nominal association in the first step of our own study for variants harbored by the same genes even if the results of the first step were not confirmed in our combined analyses. For these additional SNPs that are a common variant of $D D X 6$ and a rare variant of $I L 12 A$, we could not confirm the signal of association in our sample, ruling out independent replication. When a meta-analysis was performed, no association was observed for DDX6 rs7130875, but the original association observed for IL12A rs77583790 was strengthened, thus confirming this latter locus.

As compared with linkage analyses, association studies have much less power in cases of different mutations acting in different families; however, they are far more sensitive than family studies to study complex polygenic associations. In such diseases, the phenotype is associated with the joint affects of several weakly contributing variants across various loci. However, GWASs have received criticism and skepticism because of the structure of the knowledge it has produced, which contrasts with the highly penetrant Mendelian genetic discoveries. Indeed, interpreting and understanding the molecular mechanisms related to noncoding variants are challenging given the diversity of noncoding functions, the incomplete annotation of regulatory elements, and the probable still uncovered mechanisms of regulatory control. If some studies, through extensive experimental work, uncovered some molecular mechanisms responsible for disease association [27], far more disease-associated variants remain uncharacterized. However, expanding the catalog of non-coding variants associated with human diseases is still meaningful. Indeed, several promises have emerged; for example, the application to GWAS data of gene-set enrichment analyses has provided meaningful data regarding some regulatory mechanisms [28]. The new and complementary approaches, including integration of annotation, higher resolution, and functional maps, may provide the basis for deciphering the effects of non-coding variants in pathways or cell types taking into account the weak additive interactions discovered on GWAS and the potential epistatic interactions between the variants. Such complementary strategies might finally improve the interpretation of disease-associated variants and may help identify the most likely causal regulator. These post-genomic functional studies are eagerly awaited in order to try to elucidate the biological consequences of non-coding variants.

Herein, we identified a novel epistatic interaction between $N F-\kappa B$ and IRF8 genes, which may contribute to SSc susceptibility. This gene-gene interaction might explain the small OR obtained in our genetic association studies, which increase our understanding of missing heritability. Although the biological consequences of this interaction remain unknown, our results highlight the importance of combined analysis of genetic associations in candidate gene studies.

Taken together, our data support the shared genetic bases that are involved in SSc and PBC susceptibility. Of note, we highlight the participation of genes that are related to antigen recognition mediated by TLR and B cells, emphasizing their role in SSc susceptibility. In our mRNA expression analysis, we used resting PBMCs; hence, it is suggested that further studies use PBMC activators to enhance the expression signal.

\section{Conclusions}

Although the exact role played by PLCL2, $N F-\kappa B$, and IRF8 is yet to be determined, we provide convincing evidence which highlights the importance of these genes in SSc. Identification of new variants, interactions between unlinked loci, and epistatic effects may help us to better understand the intricate processes of heterogeneity of AIDs.

\section{Additional files}

Additional file 1: Table S1. List with detailed informations about the 16
tag SNPs included in the study. SNP - single nucleotide polymorphisms;
BP - base pairs ; variation - major allele > minor allele.
Additional file 2: Table S2. Analysis of the 13 remaining tag single-
nucleotide polymorphisms (SNPs) in the combined Caucasian populations
(French and Italian). 'After Bonferroni correction. ACA+, anti-centromere anti-
body; Cl, confidence interval; dcSSc, diffuse cutaneous systemic sclerosis;
IcSSc, limited cutaneous systemic sclerosis; MAF, minor allele frequency; n,
number of pooled patients analysed; NA, not applicable; OR, odds ratio; SSC,
systemic sclerosis; Topo I+, anti-topoisomerase I antibody.
Additional file 3: Table S3. Results of the population meta-analysis. We
used the original studies together with the French data provided in our
study and looked at single-nucleotide polymorphisms (SNPs) rs1598859,
rs7130875, rs11642873, and rs77583790.

\section{Abbreviations}

AID: autoimmune disease; Cl: confidence interval; GWAS: genome-wide association study; IFIT1: type I interferon; IFN-y: interferon-gamma; ICSSc: limited cutaneous systemic sclerosis; LD: linkage disequilibrium; MAF: minor allele 
frequency; NF-KB: nuclear factor-kappa-B; OR: odds ratio; PBC: primary biliary cirrhosis; PBMC: peripheral blood nuclear cell; PF: pulmonary fibrosis; PLC: phospholipase C; SNP: single-nucleotide polymorphism; SSc: systemic sclerosis; TGF- $\beta$ : transforming growth factor-beta; TLR: Toll-like receptor.

\section{Competing interests}

The authors declare that they have no competing interests.

\section{Authors' contributions}

MA carried out the molecular genetic experiments, statistical analysis, and interpretation of data and drafted the manuscript. MG participated in the design of the study and performed the statistical analysis and manuscript revision. NR, EK, and CK participated in the interpretation of data and manuscript revision. PD participated in DNA acquisition, the design of the study, and manuscript revision. BR carried out the molecular genetic experiments and manuscript revision. PA, DC, MM-C, ES, GC, EH, ED, PC, and VR participated in DNA and clinical data acquisition, interpretation of data, and manuscript revision. JA participated in DNA and clinical data acquisition and in manuscript revision. YA conceived the study, participated in its design and coordination, and helped to draft the manuscript and revise it. All authors read and approved the final manuscript.

\section{Acknowledgements}

We would like to thank those members of the GENESYS Consortium who contributed to the recruitment of French subjects: P. Carpentier and J. L. Cracowski in Grenoble; J. Sibilia in Strasbourg; A. Cosnes in Créteil; and J. Cabane, K. Tiev, Z. Amoura, L. Mouthon, and C. Frances in Paris. We thank J. Benessiano and B. Grandchamp (Hôpital Bichat Claude-Bernard, Paris, France) for their assistance in setting up the French Caucasian control sample. We thank the Italian centers that contributed to the control populations of HYPER- GENES study cohort in Italy. This work was funded by Association des Sclerodermies de France. We thank Pr Javier Martin, Grenada (Spain), who provided us the genotypic data from his previous studies on some SNP markers herein investigated.

\section{Author details}

${ }^{1}$ Paris Descartes University, INSERM U1016, Institut Cochin, Sorbonne Paris Cité, Paris, France. ${ }^{2}$ CAPES Foundation, Ministry of Education of Brazil, Brasília, DF 70040-020, Brazil. ${ }^{3}$ Paris Diderot University, Rheumatology Department, Hôpital Bichat Claude Bernard, APHP, Paris, France. ${ }^{4}$ Paris Diderot University, INSERM U699, Hôpital Bichat Claude Bernard, Paris, France. ${ }^{5}$ Rheumatology and Clinical Immunology, Spedali Civili, Brescia, Italy. ${ }^{6}$ University of Milano, Department of Medicine, Surgery and Dentistry San Paolo \& Genomics and Bioinformatics Platform, Fondazione Filarete, Milan, Italy. ${ }^{7}$ Department of Biomedicine \& Division of Rheumatology AOUC, Department of Rheumatology AVC, Department of Medicine \& Denothe Centre, University of Florence, Florence, Italy. ${ }^{8}$ Department of Clinical and Experimental Medicine, Rheumatology Unit, Second University of Naples, Naples, Italy. ${ }^{9}$ Université Lille II, Médecine Interne, Lille, France. ${ }^{10}$ INSERM U618, IFR 135, CHU Bretonneau, Tours, France. ${ }^{11}$ Rheumatology Unit, Azienda Ospedaliera Universitaria Integrata di Verona, Verona, Italy. ${ }^{12}$ Division of Rheumatology, Department of Medical Clinic and Therapy, University "Sapienza" of Rome, Rome, Italy. ${ }^{13}$ Paris Descartes University, Rheumatology A Department, Cochin Hospital, APHP, Paris, France. ${ }^{14}$ Department of Rheumatology, Federal University of São Paulo, São Paulo, Brazil.

Received: 4 May 2014 Accepted: 20 February 2015

Published online: 21 March 2015

\section{References}

1. Elhai M, Meune C, Avouac J, Kahan A, Allanore Y. Trends in mortality in patients with systemic sclerosis over 40 years: a systematic review and meta-analysis of cohort studies. Rheumatol Oxf Engl. 2012;51:1017-26.

2. Allanore $Y$, Dieude P, Boileau C. Updating the genetics of systemic sclerosis. Curr Opin Rheumatol. 2010;22:665-70.

3. Mayes MD. The genetics of scleroderma: looking into the postgenomic era Curr Opin Rheumatol. 2012;24:677-84.

4. Dieudé P, Guedj M, Wipff J, Ruiz B, Hachulla E, Diot E, et al. STAT4 is a genetic risk factor for systemic sclerosis having additive effects with IRF5 on disease susceptibility and related pulmonary fibrosis. Arthritis Rheum. 2009;60:2472-9.

5. Elhai M, Avouac J, Kahan A, Allanore Y. Systemic sclerosis at the crossroad of polyautoimmunity. Autoimmun Rev. 2013;12:1052-7.
6. Avouac J, Airò P, Dieude P, Caramaschi P, Tiev K, Diot E, et al. Associated autoimmune diseases in systemic sclerosis define a subset of patients with milder disease: results from 2 large cohorts of European Caucasian patients. J Rheumatol. 2010;37:608-14.

7. Mells GF, Floyd JAB, Morley KI, Cordell HJ, Franklin CS, Shin S-Y, et al. Genome-wide association study identifies 12 new susceptibility loci for primary biliary cirrhosis. Nat Genet. 2011;43:329-32.

8. Koumakis E, Giraud M, Dieudé P, Cohignac V, Cuomo G, Airò P, et al. Brief report: candidate gene study in systemic sclerosis identifies a rare and functional variant of the TNFAIP3 locus as a risk factor for polyautoimmunity. Arthritis Rheum. 2012;64:2746-52

9. Whole genome association analysis toolset available at http://pngu.mgh.harvard.edu/ purcell/plink/.

10. Martin J-E, Broen JC, Carmona FD, Teruel M, Simeon CP, Vonk MC, et al. Identification of CSK as a systemic sclerosis genetic risk factor through Genome Wide Association Study follow-up. Hum Mol Genet. 2012;21:2825-35.

11. Gorlova O, Martin J-E, Rueda B, Koeleman BPC, Ying J, Teruel M, et al. Identification of novel genetic markers associated with clinical phenotypes of systemic sclerosis through a genome-wide association strategy. PLoS Genet. 2011;7:e1002178.

12. Mayes MD, Bossini-Castillo L, Gorlova O, Martin JE, Zhou X, Chen WV, et al. Immunochip analysis identifies multiple susceptibility loci for systemic sclerosis. Am J Hum Genet. 2014;94:47-61.

13. Allanore $Y$, Saad M, Dieudé $P$, Avouac J, Distler JHW, Amouyel P, et al. Genome-wide scan identifies TNIP1, PSORS1C1, and RHOB as novel risk loci for systemic sclerosis. PLoS Genet. 2011;7:e1002091.

14. Zhao J, Kong HJ, Li H, Huang B, Yang M, Zhu C, et al. IRF-8/interferon (IFN) consensus sequence-binding protein is involved in Toll-like receptor (TLR) signaling and contributes to the cross-talk between TLR and IFN-gamma signaling pathways. J Biol Chem. 2006;281:10073-80.

15. Lafyatis R, York M. Innate immunity and inflammation in systemic sclerosis. Curr Opin Rheumatol. 2009;21:617-22.

16. Rauch I, Müller M, Decker T. The regulation of inflammation by interferons and their STATs. JAK-STAT. 2013;2:e23820.

17. Farina GA, York MR, Di Marzio M, Collins CA, Meller S, Homey B, et al. Poly(l:C) drives type I IFN- and TGF $\beta$-mediated inflammation and dermal fibrosis simulating altered gene expression in systemic sclerosis. J Invest Dermatol. 2010;130:2583-93.

18. Agarwal SK, Wu M, Livingston CK, Parks DH, Mayes MD, Arnett FC, et al. Toll-like receptor 3 upregulation by type I interferon in healthy and scleroderma dermal fibroblasts. Arthritis Res Ther. 2011;13:R3.

19. Ghosh AK, Yuan W, Mori Y, Chen SJ, Varga J. Antagonistic regulation of type I collagen gene expression by interferon-gamma and transforming growth factor-beta. Integration at the level of p300/CBP transcriptional coactivators. J Biol Chem. 2001;276:11041-8.

20. Chrabot BS, Kariuki SN, Zervou Ml, Feng X, Arrington J, Jolly M, et al. Genetic variation near IRF8 is associated with serologic and cytokine profiles in systemic lupus erythematosus and multiple sclerosis. Genes Immun. 2013;14:471-8.

21. Terao C, Ohmura K, Kawaguchi Y, Nishimoto T, Kawasaki A, Takehara K, et al. PLD4 as a novel susceptibility gene for systemic sclerosis in a Japanese population. Arthritis Rheum. 2013;65:472-80.

22. Kuryłowicz A, Nauman J. The role of nuclear factor-kappaB in the development of autoimmune diseases: a link between genes and environment. Acta Biochim Pol. 2008;55:629-47.

23. Lin C-H, Wang S-C, Ou T-T, Li R-N, Tsai W-C, Liu H-W, et al. I kappa B alpha promoter polymorphisms in patients with systemic lupus erythematosus. J Clin Immunol. 2008;28:207-13.

24. Takenaka K, Fukami K, Otsuki M, Nakamura Y, Kataoka Y, Wada M, et al. Role of phospholipase C-L2, a novel phospholipase C-like protein that lacks lipase activity, in B-cell receptor signaling. Mol Cell Biol. 2003;23:7329-38.

25. Raychaudhuri S, Thomson BP, Remmers EF, Eyre S, Hinks A, Guiducci C, et al. Genetic variants at CD28, PRDM1 and CD2/CD58 are associated with rheumatoid arthritis risk. Nat Genet. 2009;41:1313-8.

26. Bowes J, Ho P, Flynn E, Ali F, Marzo-Ortega H, Coates LC, et al. Comprehensive assessment of rheumatoid arthritis susceptibility loci in a large psoriatic arthritis cohort. Ann Rheum Dis. 2012;71:1350-4.

27. Sakabe NJ, Savic D, Nobrega MA. Transcriptional enhancers in development and disease. Genome Biol. 2012;13:238.

28. Ernst J, Kheradpour P, Mikkelsen TS, Shoresh N, Ward LD, Epstein CB, et al. Mapping and analysis of chromatin state dynamics in nine human cell types. Nature. 2011;473:43-9. 